\title{
Recuperación estructural y funcional de los espacios entre arbustos al cabo de 10 años de exclusión del pastoreo en una estepa semiárida del noreste de la Patagonia
}

\author{
Flavia A. FunK ${ }^{1, \otimes} ;$ GuAdalupe Peter $^{2,3}$; Alejandro Loydi ${ }^{1}$; Alicta I. KRÖPfl ${ }^{2} \&$ Roberto A. Distel ${ }^{1,4}$ \\ 1. CERZOS, CCT CONICET Bahía Blanca. Bahía Blanca, Argentina. 2. CURZA, Universidad Nacional del Comahue. \\ Viedma, Argentina. 3. Sede Atlántica, Universidad Nacional de Río Negro. Viedma, Argentina. 4. Departamento de \\ Agronomía, Universidad Nacional del Sur. Bahía Blanca, Argentina.
}

\begin{abstract}
RESUMEN. En las estepas arbustivas del noreste de la Región Patagónica, el sobrepastoreo del ganado doméstico genera la formación de islas arbustivas distribuidas en una matriz degradada de suelo desnudo o con escasa cobertura vegetal. La recuperación estructural y funcional de los espacios entre arbustos mediante la remoción del ganado dependería de la magnitud de la degradación. Si el sistema ha atravesado un umbral crítico de degradación, la sola remoción del disturbio no sería suficiente para revertir las alteraciones provocadas por el pastoreo. El objetivo del trabajo fue evaluar la recuperación de espacios entre arbustos en un sitio representativo del sistema en estudio luego de 10 años de exclusión del pastoreo, mediante la comparación de la cobertura y propiedades físicoquímicas del suelo entre áreas clausuradas y pastoreadas. Se realizaron mediciones en cuatro áreas clausuradas y en áreas aledañas sometidas a pastoreo continuo. Se comparó la cobertura (vegetación, pavimento de erosión, costra biológica, suelo desnudo y broza), el tamaño de los espacios entre arbustos, la tasa de infiltración, la textura y el contenido de nutrientes del suelo. La cobertura aérea de gramíneas perennes y de costras biológicas fue mayor en las áreas clausuradas ( $27 \%$ y $45 \%$, respectivamente) que en las pastoreadas $(<1 \%$ y $3 \%)$. El tamaño de los espacios entre arbustos fue mayor en las áreas pastoreadas que en las clausuradas. La tasa de infiltración y las propiedades físicoquímicas del suelo no difirieron entre las condiciones de clausura y pastoreo, aunque la densidad aparente y el contenido de arena en la capa superficial del suelo fueron ligeramente mayores ( $7 \%$ y $3 \%$, respectivamente) en las áreas pastoreadas. La exclusión del pastoreo por un período de 10 años resultó en el restablecimiento de gramíneas perennes y costras biológicas, sugiriendo que la alteración estructural y funcional de los espacios entre arbustos aún no ha superado un umbral crítico de degradación.
\end{abstract}

[Palabras clave: desertificación, degradación de pastizales, gramíneas perennes, infiltración, densidad aparente, fertilidad del suelo]

\begin{abstract}
AвSTRACT. Structural and functional recovery of intershrub spaces after 10-years of grazing exclusion in a semiarid steppe of northeastern Patagonia: In shrubby steppes of northeastern Patagonia, overgrazing of domestic livestock produces the formation of shrub islands distributed in a degraded matrix of bare soil or sparce vegetation cover. The structural and functional recovery of intershrub spaces through livestock removal would depend on the magnitude of degradation. If the system has crossed a critical threshold of degradation, the sole removal of the disturbance would be not sufficient to reverse the changes caused by grazing. The objective of this study was to assess the recovery of intershrub spaces in a site representative of the study system after 10 years of livestock grazing exclusion, by comparing soil coverage and soil physicochemical properties between ungrazed and grazed areas. Data sets were collected in four plots excluded from grazing and in areas adjacent to each plot that remained under continuous grazing. We measured the size of the intershrub spaces, and soil coverage (vegetation, erosion pavement, biological soil crusts, litter and bare soil), texture, nutrient content and infiltration rate. We found that the aerial coverage of perennial grasses and biological soil crusts was higher in ungrazed areas ( $27 \%$ and $45 \%$, respectively) than in grazed areas $(<1 \%$ and $3 \%$, respectively). The size of the intershrub spaces was higher in grazed areas than in ungrazed areas. The physicochemical properties of soil and infiltration rate did not differ between ungrazed and grazed areas, except that bulk density and sand content in the soil-surface layers were slightly higher (7\% and $3 \%$, respectively) in grazed areas. The sole exclusion of grazing for a period of 10 years allowed the recovery of perennial grasses and biological crusts, suggesting that the structural and functional alterations of the intershrub spaces has not yet exceeded a critical threshold of degradation.
\end{abstract}

[Keywords: desertification, rangeland degradation, perennial grasses, infiltration, bulk density, soil fertility]

\section{INTRODUCCIÓN}

En los ambientes áridos y semiáridos, el sobrepastoreo del ganado doméstico es el principal factor de desertificación al transformar ambientes dominados por gramíneas perennes en ambientes dominados por arbustos con suelo desnudo o baja cobertura vegetal en los espacios entre arbustos (Milchunas \& Lauenroth 1993; 
Whitford 1997; Allington \& Vallone 2011). Las modificaciones estructurales mencionadas comúnmente alteran procesos fundamentales de los ecosistemas, tales como el ciclado de nutrientes, el flujo de agua y la productividad primaria (Reynolds et al. 1999). En general, se propone que el disturbio del ganado puede ser el disparador de alteraciones persistentes en la hidrología del suelo (e.g., la disminución de la tasa de infiltración y el aumento de la escorrentía), y convertiría en disfuncionales a los ecosistemas en términos de eficiencia de uso del agua, nutrientes y radiación (Bestelmeyer et al. 2004; Briske et al. 2005). Esta reducción en la disponibilidad de nutrientes y en las propiedades físicas del suelo es provocada por la remoción de gramíneas palatables y por el pisoteo del ganado; esto limita el establecimiento y el desarrollo de nuevas plántulas (Bisigato \& Bertiller 2004).

Cuando las alteraciones estructurales y funcionales superan un umbral crítico de degradación, el sistema cambiaría a un estado estable alternativo y se genera una transición irreversible en términos de control del disturbio (Walker et al. 1981; Le Houerou 1989; Bestelmeyer et al. 2004). Así, cuando en los ambientes áridos y semiáridos un pastizal se convierte en arbustal, la reversión de la transición no podría lograrse a corto plazo sin una intervención mucho mayor que el simple control del disturbio (Westoby et al. 1989; Laycock 1991; Valone et al. 2002; Stringham et al. 2003). La disminución de la cobertura de las gramíneas favorecería la compactación del suelo, lo que reduce la tasa de infiltración del agua e incrementa la erosión del suelo (Rostagno 1989). Este mecanismo de degradación mantiene o refuerza las comunidades vegetales degradadas y limita el retorno hacia la comunidad original (Scheffer et al. 2001; van de Koppel et al. 2002). No obstante, resultados recientes sugieren que la exclusión del pastoreo podría disparar la recuperación de la vegetación y de las propiedades del suelo en los espacios entre arbustos (Fuhlendorf et al. 2001; Valone et al. 2002; Zhang et al. 2005). Con el paso del tiempo la compactación del suelo disminuiría; esto favorecería la infiltración del agua y el restablecimiento de vegetación (Castellano \& Valone 2007; Allington \& Vallone 2011). Dicha recuperación del suelo estaría influenciado por el régimen de precipitaciones. Por lo tanto, la alta variación interanual de la precipitación de los ambientes áridos probablemente interactúe con la tasa de infiltración y retarde la recuperación de la vegetación (Castellano \&
Valone 2007). Debido a que el agua constituye el principal factor limitante para el crecimiento de las plantas en los sistemas áridos y semiáridos, el aumento de la tasa de infiltración del agua en el suelo resultaría esencial para la recuperación del sistema (Walker et al. 1981). Más aún, el establecimiento de plantas promovería la infiltración del agua en el suelo, y se produciría una retroalimentación positiva entre cobertura vegetal y tasa de infiltración (van de Koppel et al. 2002).

En el noreste de la Patagonia argentina, el sobrepastoreo por ganado doméstico genera la formación de islas arbustivas distribuidas en una matriz degradada de suelo desnudo o con escasa cobertura vegetal (espacios entre arbustos) (Bisigato \& Bertiller 1997). El disturbio por pastoreo ha provocado una reducción fuerte de la cobertura de gramíneas perennes, el aumento de la erosión hídrica y eólica en los parches de suelo desnudo, y el deterioro físicoquímico de los suelos (Rostagno \& del Valle 1988; Bisigato \& Bertiller 1997; Kröpfl 1999). Son escasos los antecedentes regionales acerca de la potencial recuperación de la vegetación y propiedades del suelo en los espacios entre arbustos en respuesta a la exclusión del pastoreo (Bisigato et al. 2002). El objetivo del presente trabajo fue evaluar la recuperación de espacios entre arbustos en un sitio representativo del sistema en estudio luego de 10 años de exclusión del pastoreo, mediante la comparación de la cobertura y las propiedades físicoquímicas del suelo entre áreas clausuradas y áreas pastoreadas. Dado que la limitante principal para la recuperación del ambiente estaría dada por la disponibilidad de agua, esperamos que al retirar el ganado (10 años de exclusión) la compactación del suelo producida por el pisoteo disminuya y -por lo tanto- aumente la tasa de infiltración del agua. Este aumento en la infiltración facilitaría el establecimiento de las gramíneas perennes y el incremento de la concentración de nutrientes del suelo en los espacios entre arbustos.

\section{Materiales y MÉTOdos}

El estudio se llevó a cabo en el noreste de la Provincia de Río Negro, Argentina $\left(40^{\circ} 40^{\prime} \mathrm{S}\right.$, $64^{\circ} 10^{\prime} \mathrm{O}$ ). Desde fines del siglo XIX, la actividad económica predominante en la zona ha sido la ganadería ovina, aunque a partir de la segunda mitad del siglo XX adquirió cierta preponderancia la ganadería bovina (Ares et al. 1990). El clima de la región es semiárido templado. La temperatura media anual del aire es $14^{\circ} \mathrm{C}$, con temperaturas medias mensuales que varían entre $7^{\circ} \mathrm{C}$ en julio 
y $21{ }^{\circ} \mathrm{C}$ en enero. Las precipitaciones promedian los $270 \mathrm{~mm} /$ año $(\mathrm{CV}=40 \%)$. Las lluvias más abundantes tienen lugar en otoño y primavera. La evapotranspiración potencial anual ronda los $800 \mathrm{~mm}$. Se registran vientos durante todo el año, aunque con mayor intensidad en primavera y verano, lo que contribuye a hacer más marcado el déficit hídrico. Los suelos dominantes en sectores planos se clasifican como Natrargides ustólico, y en los microrelieves de loma como Haplocambidefluventico (Godagnone \& Bran 2009). Fisonómicamente, la vegetación conforma una estepa arbustivo-graminosa baja y corresponde a la Provincia Fitogeográfica del Monte (Cabrera 1971). La vegetación se distribuye en "islas" dominadas por arbustos (uno o más individuos), rodeadas por suelo desnudo o con escasa cobertura vegetal. Las especies dominantes del estrato arbustivo son Chuquiraga erinacea D. Don, Larrea divaricata Cav., L. cuneifolia Cav., Condalia microphylla Cav., Prosopis alpataco Phil., Senna aphylla (Cav.) H.S. Irwin \& Barneby, Lycium chilense Miers, L. tenuispinosum Miers, Monttea aphylla (Miers) Benth. \& Hook., Schinus johnstonii F.A. Barkley y Ephedra ochreata Miers. En el estrato herbáceo de las islas arbustivas y de los espacios entre islas están presentes las gramíneas perennes nativas Nasella tenuis (Phil.) Barkworth, N. longiglumis (Phil.) Barkworth, Poa ligularis Nees. ex Steud., Piptochaetium napostaense (Speg.) Hack., Jarava plumosa (Spreng.) S.W.L. Jacobs \& J. Everett y Pappostipa speciosa (Trin. \& Rupr.) Romasch. y especies anuales exóticas tales como Bromus catharticus Vahl., Schismus barbatus (L.) Thell. y Erodium cicutarium (L.) L'Hér. ex Aiton (Cabrera 1971). La costra biológica (comunidades de microorganismos como algas, hongos, líquenes, musgos y cianobacterias que se encuentran íntimamente interrelacionados) está dominada por dos especies de musgos: Syntrichia princeps (De Not.) Mitt. y Ceratodon purpureus (Hedw.) Brid. (Kröpfl et al. 2011).

La ubicación del sitio de estudio se eligió de modo tal que resultara lo más representativa posible de la estepa arbustivo-graminosa del noreste del Monte rionegrino, en términos de suelo-topografía y vegetación. El sitio de estudio acredita 100 años de historia de pastoreo continuo por ganado ovino, con un nivel de carga usual para la región $(0.36$ $\mathrm{EO} / \mathrm{ha}$ ). Las mediciones se realizaron en cuatro áreas de 20×10 m, excluidas del pastoreo ovino por un alambrado a mediados del año 2000 (áreas clausuradas), y en cuatro áreas de las mismas dimensiones y apareadas con las anteriores, que continuaron de manera ininterrumpida bajo pastoreo ovino (áreas pastoreadas) (ver fotografía en información suplementaria). Los cuatro pares estaban separados entre sí por una distancia de $\sim 20 \mathrm{~m}$ y distanciados $900 \mathrm{~m}$ del abrevadero más cercano. Las precipitaciones anuales durante el periodo de estudio se obtuvieron de una estación meteorológica ubicada en San Antonio Oeste, a $80 \mathrm{~km}$ de distancia del sitio de estudio. Durante la primera mitad del período de exclusión del pastoreo (2000-2005) las precipitaciones anuales superaron al promedio histórico o fueron levemente inferiores (2003 y 2005), mientras que en la segunda mitad fueron menores al promedio histórico (representaron entre 35\% y 75\% de la precipitación anual promedio histórica), a excepción del año 2006, en el que fueron similares.

Se evaluó la cobertura y las propiedades fisicoquímicas del suelo en los espacios entre arbustos entre agosto de 2010 y noviembre de 2011. La fecha fue seleccionada entre los picos de lluvia a fin de evitar variaciones importantes en el contenido inicial de humedad del suelo.

La cobertura aérea de la vegetación se determinó a nivel de especie. Se utilizaron parcelas de muestreo de $1 \times 1 \mathrm{~m}$ ( $\mathrm{n}=5$ por área, $\mathrm{N}=40)$, ubicadas en espacios entre arbustos elegidos al azar dentro de cada área clausurada y en el área pastoreada aledaña correspondiente. La cobertura se estimó en forma visual empleando la escala de coberturas de Braun-Blanquet. La cobertura de pavimento de erosión (presencia de canto rodado en la superficie), costra biológica, suelo desnudo y broza se determinó de igual forma que la cobertura aérea de la vegetación, pero utilizando parcelas de $0.25 \times 0.25 \mathrm{~m}$ subdivididas en 100 cuadrículas $(n=15$ por área, $\mathrm{N}=120$ ). Dichas parcelas se ubicaron al azar entre matas de gramíneas perennes en los espacios entre arbustos. La distancia promedio entre islas arbustivas, tomada como subrogante del tamaño de los espacios entre islas, se determinó sobre transectas lineales de $10 \mathrm{~m}$ de longitud ( $\mathrm{n}=2$ por área, $\mathrm{N}=16)$ ubicadas al azar en las áreas clausuradas y en las pastoreadas. Se midieron las distancias entre islas interceptadas por la transecta. Se definió como "isla" al área dominada por arbustos (incluyendo el área debajo de la proyección del canopeo al suelo), que por lo general presenta una acumulación de sedimento visiblemente mayor al área circundante.

Las mediciones de infiltración del agua en el suelo de los espacios entre arbustos se efectuaron entre matas de vegetación herbácea evitando disturbar la superficie del suelo, en dos oportunidades: agosto de 2010 y noviembre de 2011. En la primera fecha de medición se emplearon infiltrómetros de doble anillo (Anillo de Munz, con diámetro del anillo interno y externo de 30 y $45 \mathrm{~cm}$, respectivamente; $\mathrm{n}=1$ por área, $\mathrm{N}=8$ ), mientras que en la segunda fecha de medición se emplearon infiltrómetros de anillos simples (32 cm de diámetro; $\mathrm{n}=2$ por área, $\mathrm{N}=16$ ). Ambos tipos de infiltrómetros suministran mediciones comparables (Johnson 1991; Verbist et al. 2010). Además, en el presente estudio el interés primario fue la comparación de la tasa de infiltración básica entre las áreas clausuradas y las pastoreadas. Para las comparaciones entre ambos tipos de áreas se utilizó la metodología habitual (ver Sepúlveda (1999) para una descripción detallada de las técnicas de infiltración). En ambas fechas, todas las mediciones se realizaron durante el mismo día. Se efectuaron tres lecturas por cada intervalo de tiempo considerado: 5, 10, 20 y 30 minutos (12 
lecturas en total). Además se tomaron muestras de suelo ( $\mathrm{n}=5$ por área, $\mathrm{N}=40$ ) para determinar el contenido de agua por gravimetría, con el fin de comparar el estado hídrico del suelo entre ambos regímenes de pastoreo.

La caracterización del suelo de los espacios entre arbustos se realizó mediante determinaciones de textura, densidad aparente y del contenido de materia orgánica, nitrógeno total, fósforo extractable. Las muestras de suelo fueron recolectadas en lugares elegidos al azar en marzo de 2011. Para los análisis de textura se tomaron muestras compuestas (cada una integrada por cinco submuestras provenientes de espacios entre arbustos) de los primeros $5 \mathrm{~cm}$ del suelo ( $\mathrm{n}=2$ por área, $\mathrm{N}=16$ ), separando la costra física superficial del suelo subyacente. Dicha costra física es la capa superficial de suelo, comúnmente de menos de $1 \mathrm{~cm}$ de espesor, estructuralmente diferente al material que se encuentra inmediatamente debajo de ellas (Belnap et al. 2001). En ambas fracciones de suelo se determinó el porcentaje de arcilla, limo y arena por el método del densímetro de Bouyoucos. Para la determinación de la densidad aparente se tomaron muestras de suelo sin disturbar $(n=5$ por área, $\mathrm{N}=40$ ) empleando cilindros de acero de $5 \mathrm{~cm}$ de diámetro por $5 \mathrm{~cm}$ de profundidad (100 $\mathrm{cm}^{3}$ ), luego fueron secadas a $100^{\circ} \mathrm{C}$ en estufa y se determinó la relación peso/volumen. Para los análisis químicos se tomaron muestras compuestas (misma metodología que para el análisis de textura) de los primeros $5 \mathrm{~cm}$ de suelo $(\mathrm{n}=1$ por área, $\mathrm{N}=8$ ). La materia orgánica se obtuvo por combustión seca mediante un Analizador Automático de Carbono (LECO modelo CR12) y utilizando el factor de corrección de van Bemmelen $(\% \mathrm{MO}=\% \mathrm{C} * 1.724)$, el $\mathrm{N}$ total por el método semimicro Kjeldahl, y el $\mathrm{P}$ extractable por el método de Bray-Kurtz.

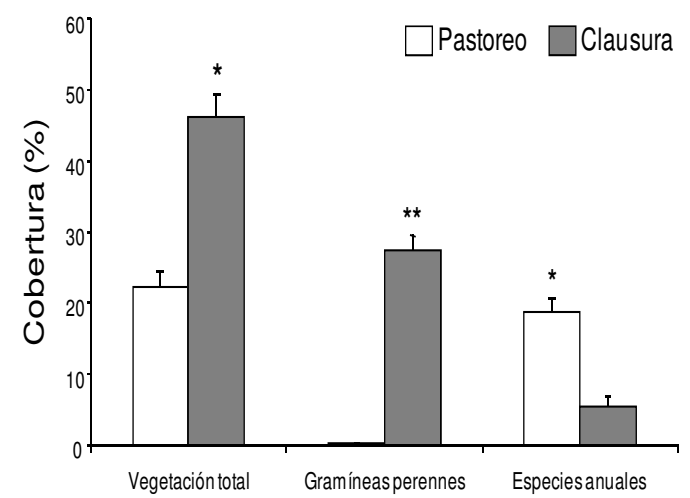

Figura 1. Cobertura media de la vegetación total, gramíneas perennes y especies anuales en las áreas pastoreadas (AP) y clausuradas (AC) (10 años de antigüedad). Las líneas verticales sobre las barras indican el ES. ${ }^{*} P<0.05 y * * P<0.01$.

Figure 1. Average cover of the total vegetation, perennial grasses and annual species on grazed (AP) and ungrazed areas (AC) (10 years excluded). The vertical lines above the bars indicate the SE. ${ }^{*} P<0.05$ and ${ }^{* *} P<0.01$.

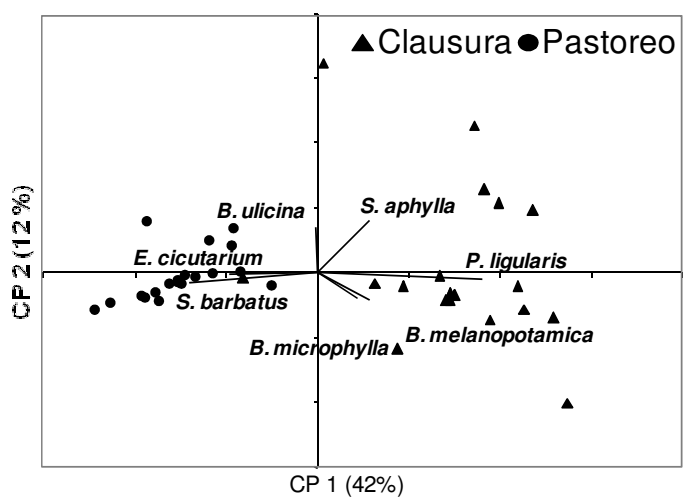

Figura 2. Ordenación de las áreas pastoreadas y clausuradas (10 años de antigüedad) por composición florística en los espacios entre arbustos mediante análisis de componentes principales. CP: componente principal, los valores entre paréntesis indican la varianza explicada por cada eje.

Figure 2. Ordination of grazed and ungrazed (10 years excluded) areas by floristic composition in intershrub spaces through principal components analysis. CP: principals components, the values in parentheses indicate the explained variance for each axis.

Los datos obtenidos se analizaron mediante pruebas t de Student para muestras apareadas, excepto la cobertura de gramíneas perennes. Esta última variable se analizó con la prueba de Wilcoxon (no paramétrica), debido a que los datos no seguían una distribución normal. Previo análisis, los datos de cobertura de especies anuales, suelo desnudo y de pavimento de erosión fueron transformados al arcoseno de su raíz para cumplir el supuesto de normalidad, si bien los resultados se muestran sin transformar. Para la ordenación de los sitios de acuerdo a su composición florística se utilizó un análisis de componentes principales empleando matrices de covarianza. Los valores de la tasa de infiltración básica fueron obtenidos mediante el modelo de Kostiakov (Hillel 1998). Todos los análisis se realizaron mediante el programa InfoStat (Di Renzo et al. 2009).

\section{RESULTADOS}

La cobertura vegetal total en los espacios entre arbustos fue mayor $(P<0.05)$ en las áreas clausuradas que en las pastoreadas, al igual que la cobertura de gramíneas perennes $(P<0.01)(27 \%$ y $0.17 \%$, respectivamente). En cambio, la cobertura de especies anuales fue mayor $(P<0.05)$ en las áreas pastoreadas (Figura 1). Los dos primeros componentes principales explicaron $56 \%$ de la varianza total. El primer componente principal diferenció a ambos tipos de áreas por la identidad de las especies dominantes, ya que las áreas clausuradas se caracterizaron por la dominancia de $P$. ligularis, mientras que las áreas pastoreadas se caracterizaron por la dominancia de $S$. barbatus y E. cicutarium. 
Tabla 1. Valores (media \pm 1 ES) de cobertura en espacios entre arbustos y distancia entre islas arbustivas bajo condiciones de pastoreo y clausura (10 años de antigüedad).

Table 1. Cover values (mean $\pm 1 \mathrm{SE}$ ) in intershrub spaces and distance between shrub islands under grazed and ungrazed (10 years excluded) conditions.

\begin{tabular}{lccc}
\hline Variables & Pastoreo & Clausura & $\mathrm{P}$ \\
\hline Cobertura (\%) & & & \\
Pavimento de erosión & $57.95 \pm 1.47$ & $12.08 \pm 2.49$ & $<0.01$ \\
Costra biológica & $3.05 \pm 0.22$ & $45.41 \pm 2.57$ & 0.01 \\
Suelo desnudo & $32.16 \pm 1.35$ & $34.74 \pm 3.15$ & 0.29 \\
Broza & $6.83 \pm 0.19$ & $7.76 \pm 0.97$ & 0.41 \\
Distancia entre islas (m) & $5.27 \pm 0.44$ & $2.74 \pm 0.42$ & 0.05 \\
\hline
\end{tabular}

El segundo componente principal separó las parcelas excluidas del pastoreo de acuerdo a la identidad de los subarbustos presentes en las mismas. En un grupo de parcelas predominaron Baccharis ulicina Hook. \& Arn. y S. aphylla, y en otro grupo Baccharis melanopotamica Speg. y Bredemeyera microphylla (Griseb.) Hieron (Figura 2).

La cobertura de pavimento de erosión fue mayor $(P<0.01)$ en las áreas pastoreadas mientras que la cobertura de costras biológicas fue mayor $(P<0.01)$ en las áreas clausuradas (Tabla 1). La cobertura de suelo desnudo y de broza fue similar en ambas condiciones (pastoreo y clausura). La distancia promedio entre islas arbustivas fue mayor $(P<0.05)$ en las áreas pastoreadas que en las clausuradas.

Las tasas de infiltración básica registradas en las condiciones de clausura y pastoreo resultaron similares $(P>0.05)$, tanto cuando las mediciones se realizaron con infiltrómetros de doble anillo (media \pm EE: $54.46 \pm 18.06 \mathrm{~mm}$ / h y $33.03 \pm 8.9 \mathrm{~mm} / \mathrm{h}$, respectivamente), como cuando se realizaron con infiltrómetros de anillo simple (media \pm EE: $32.76 \pm 2.88 \mathrm{~mm} /$ h y $33.45 \pm 2.85 \mathrm{~mm} / \mathrm{h}$, respectivamente). Al momento de realizar las mediciones de infiltración, la humedad gravimétrica promedio del suelo fue $4 \%$ (agosto 2010) y $8 \%$ (noviembre 2011), sin diferenciarse $(P>0.05)$ entre las condiciones de pastoreo y clausura en ambas fechas.

No se observaron diferencias texturales en los espacios entre arbustos entre ambos tipos de áreas, excepto que la costra superficial fue más arcillosa $(P<0.05)$ en las áreas clausuradas y más arenosa en las pastoreadas (Tabla 2). La densidad aparentefue $7 \%$ menor $(P<0.05)$ en las áreas clausuradas. Las propiedades químicas del suelo no difirieron significativamente entre ambas condiciones $(P>0.05)$, aunque en términos de valores absolutos fueron consistentemente superiores $(\sim 12 \%)$ en las áreas clausuradas (Tabla 2).

\section{DisCUSIÓN}

Los resultados obtenidos pusieron de manifiesto cierto grado de recuperación de la vegetación herbácea en los espacios entre arbustos al cabo de 10 años de exclusión del pastoreo. Dicha respuesta no tuvo correlato con una mejora en las propiedades fisicoquímicas del suelo, que permanecieron sin cambios significativos durante el período de estudio, excepto por una disminución en la densidad aparente del suelo en las áreas clausuradas al pastoreo. El restablecimiento de gramíneas perennes y de costra biológica en las áreas clausuradas resulta particularmente significativo, más aún si se considera la escasez de precipitaciones durante la segunda mitad del período de exclusión al pastoreo. En regiones áridas y semiáridas de otras partes del mundo, la recuperación de la vegetación herbácea en áreas excluidas al pastoreo tardó entre 20 y 40 años, a diferencia del caso en estudio, y estuvo acompañada de mejoras en las propiedades fisicoquímicas del suelo (e.g., el aumento en la tasa de infiltración del agua, la concentración de nutrientes y la disminución en la compactación del suelo) (Valone et al. 2002; Castellano \& Valone 2007; Seymour et al. 2010; Allington \& Valone 2010, 2011).

La recuperación de la cobertura de gramíneas perennes en los espacios entre arbustos en respuesta a la exclusión al pastoreo podría estar explicada por un efecto facilitador de las costras biológicas (Bowker 2007), en particular por actuar como trampa de semilla y proveer de sitios seguros para el establecimiento de plántulas (Evans \& Johansen 1999). Las costras biológicas pueden recuperarse en pocos años en ausencia de pastoreo (Read et al. 2011), y

Tabla 2. Valores (media \pm 1 ES) de propiedades fisicoquímicas del suelo en espacios entre arbustos bajo condiciones de pastoreo (AP) y clausura (AC) (10 años de antigüedad).

Table 2. Values (mean \pm 1 SE) of soil physicochemical properties in intershrub spaces under grazed (AP) and ungrazed (AC) (10 years excluded) conditions.

\begin{tabular}{lccr}
\hline Variables & Pastoreo & Clausura & P \\
\hline Costras físicas & & & \\
Arena (\%) & $63 \pm 1.73$ & $60 \pm 0.81$ & 0.05 \\
Limo (\%) & $20.25 \pm 0.63$ & $20.50 \pm 1.26$ & 0.35 \\
$\quad$ Arcilla (\%) & $16.25 \pm 1.43$ & $19.50 \pm 0.50$ & 0.05 \\
Suelo & & & \\
Arena (\%) & $63 \pm 1.73$ & $63 \pm 1.30$ & 0.50 \\
Limo (\%) & $18.50 \pm 0.50$ & $20 \pm 0.81$ & 0.10 \\
Arcilla (\%) & $18.50 \pm 1.90$ & $17 \pm 1.30$ & 0.10 \\
Densidad aparente $\left(\mathrm{g} / \mathrm{cm}^{3}\right)$ & $1.36 \pm 0.02$ & $1.27 \pm 0.04$ & $<0.01$ \\
Materia orgánica $(\%)$ & $1.36 \pm 0.26$ & $1.55 \pm 0.09$ & 0.29 \\
Nitrógeno total $(\%)$ & $0.06 \pm 0.01$ & $0.07 \pm 0.003$ & 0.31 \\
Fósforo extractable $(\mathrm{ppm})$ & $15.15 \pm 1.90$ & $18.07 \pm 3.41$ & 0.30 \\
\hline
\end{tabular}


favorecerían el establecimiento de gramíneas perennes en los espacios entre arbustos en sistemas similares al estudiado (Bertiller \& Ares 2011). La recuperación observada también podría deberse en parte a mejoras de las propiedades del suelo a una escala mucho menor del microrrelieve, es decir, asociadas a las matas de gramíneas. Debido a la localización de los muestreos de suelo y de las mediciones de infiltración en los espacios entre matas de pastos, los cambios en las propiedades edáficas pudieron no ser detectados en la escala a la que fueron medidos. Dicho procedimiento tuvo por fin estandarizar las comparaciones entre ambos tipos de condiciones. Sin embargo, es de esperar que el restablecimiento de vegetación en los espacios entre arbustos genere en la proximidad de las plantas establecidas una retroalimentación positiva con la fertilidad del suelo y con la tasa de infiltración del agua en el mismo (Thurow et al. 1988; Tongway et al. 2003).

Entre las gramíneas perennes que se restablecieron en los espacios entre arbustos de las áreas clausuradas cabe destacar la preponderancia de $P$. ligularis, ya que se trata de una especie que posee una palatabilidad alta para el ganado, y cuya cobertura decrece a medida que aumenta la intensidad del pastoreo, en coincidencia con un aumento de especies más xerófilas (Perelman et al.1997; Bertiller \& Bisigato 1998). La recuperación de esta especie está posiblemente relacionada con el rol de refugio biótico que cumplen las islas arbustivas en los ambientes áridos y semiáridos (Milchunas \& Noy-Meir 2002), como ha quedado demostrado en el Monte Oriental Patagónico (Pazos \& Bertiller 2008). Las plantas que sobreviven el pastoreo mediante la protección de los arbustos proveen propágulos para el reclutamiento de individuos en los ambientes que fueron disturbados.

En las áreas pastoreadas las islas arbustivas se encontraron más distanciadas entre sí que en sus pares clausurados, lo cual determinaría un mayor tamaño de los espacios entre arbustos en el primer caso. Esta observación fue consistente con resultados obtenidos en otros trabajos realizados en el Monte Patagónico, donde se observó que el tamaño de los espacios entre islas arbustivas aumenta con la intensidad del pastoreo (Bisigato \& Bertiller 1997; Bisigato et al. 2005). El mayor tamaño de los espacios entre islas en las áreas pastoreadas podría atribuirse a la pérdida de arbustos, al menor crecimiento de los individuos presentes y/o a una disminución del reclutamiento de las especies arbustivas bajo condiciones de pastoreo severo, y/o a la proliferación del estrato arbustivo en las áreas clausuradas. Algunos trabajos recientes han puesto de manifiesto que la exclusión del pastoreo doméstico puede favorecer la proliferación de las especies leñosas (i.e., más cobertura, más densidad), en comparación con áreas aledañas a las clausuras que permanecieron bajo pastoreo (Browning \& Archer 2011; Cesa \& Paruelo 2011; Cipriotti \& Aguiar 2012).

La similitud entre las áreas clausuradas y las pastoreadas en la tasa de infiltración y en las propiedades fisicoquímicas del suelo medidas en los espacios entre matas de pastos coincidió con resultados informados por Castellano \& Valone (2007) para una estepa semiárida (sudoeste de los Estados Unidos) con similar tiempo de exclusión al pastoreo (10 años). Las mejoras en la infiltración y en las propiedades fisicoquímicas del suelo en los espacios entre matas se han observado comúnmente luego de un mayor tiempo de exclusión al pastoreo ( $\geq 20$ años) (Drewry 2006; Steffens et al. 2008; Allington \& Valone 2010, 2011). De todos modos, vale destacar la disminución de la densidad aparente del suelo y el mayor valor absoluto de las propiedades químicas del suelo en los espacios entre arbustos de las áreas clausuradas al pastoreo en comparación con sus pares pastoreadas. No obstante, el tiempo de exclusión al pastoreo requerido para la recuperación de las propiedades fisicoquímicas del suelo es de esperar varíe con el clima, el suelo, el tipo de vegetación y la historia de pastoreo previa a su exclusión (Castellano \& Valone 2007; Bisigato et al. 2008).

El disturbio de los pastizales por pastoreo (en interacción con sequía y fuego) comúnmente genera estados estables alternativos (Westoby et al. 1989; Briske et al. 2005). La transición entre estados no es abrupta sino que se irían atravesando umbrales de distinto tipo (patrón, proceso, degradación) (sensu Bestelmeyer 2006). Una vez atravesado un umbral de degradación, la sola eliminación del disturbio no resultaría suficiente para la reversión a un estado original (Stringham et al. 2003). En el noreste de la Región Patagónica, la estepa arbustivo-graminosa actual, caracterizada por la formación de islas arbustivas dispersas en una matriz con escasa cobertura vegetal (espacios entre arbustos), constituiría un estado estable que habría derivado de la 
estepa graminosa-arbustiva prístina (Bertiller \& Bisigato 1998). Numerosos trabajos han propuesto que la sola exclusión del pastoreo no sería suficiente para revertir los cambios estructurales y funcionales de la vegetación, debido en particular a la estabilidad elevada del componente arbustivo (Walker et al. 1981; Westoby et al. 1989; Laycock 1991; Valone et al. 2002). Sin embargo, de acuerdo a nuestros resultados, la recuperación de los pastos y de la costra biológica en los espacios entre arbustos luego de 10 años de exclusión del pastoreo sugiere que la degradación de estos ambientes es potencialmente reversible si se suprime el disturbio. Cabe destacar que en este trabajo no se han examinado de forma directa los umbrales de degradación del sistema, los cuales -por lo general- son difíciles de determinar (Radford et al. 2005; Bestelmeyer 2006), así como tampoco la recuperación bajo diferentes historias de uso de la tierra. No obstante, bajo ciertas condiciones, el control del pastoreo representaría una herramienta útil para la recuperación de las estepas arbustivograminosas del noreste de la Patagonia.

Agradecimientos: Agradecemos al Sr. Griffit O. por permitirnos el acceso al área de estudio. Además a Leder C., Torres Robles S., Zeberio J.M. y Luna M. por su colaboración en el trabajo de campo. A Martínez S. por su asesoramiento y préstamo de equipos. Y a dos revisores anónimos por sus comentarios en el manuscrito. Este trabajo fue subsidiado con un proyecto de CONICET (PIP 112-200801-01046).

\section{BiBLIOGRAFÍA}

Allington, GRH \& TJ Valone. 2010. Reversal of desertification: the role of physical and chemical soil properties. J. Arid. Environ., 74:973-977.

AlLinGton, GRH \& TJ Valone. 2011. Long-term livestock exclusion in an arid grassland alters vegetation and soil. Rangeland Ecol. Manag., 64:424-428.

Ares, J; AM Beeskow; MB Bertiller; CM Rostagno; MP IRISARRI; ET AL. 1990. Structural and dynamic characteristics of overgrazed lands of northern Patagonia, Argentina. Pp. 149-175 en: Breymeyer, A (ed.). Managed Grasslands: regional studies. Vol. 17 A. Elsevier. Amsterdam. Pp. 387.

Belnap, J; R Rosentreter; S Leonard; JH Kaltnecker; J WiLliams; ET AL. 2001. Biological soil crusts: ecology and management. Tech. Ref. 1730-2, US Department of Interior Bureau of Land Management. Denver, Co.

Bertiller, MB \& JO Ares. 2011. Does sheep selectivity along grazing paths negatively affect biological crusts and soil seed banks in arid shrublands? A case study in the Patagonian Monte, Argentina. J. Environ. Manage., 92:2091-2096.

BERTILLER, MB \& A BisIGATO. 1998. Vegetation dynamics under grazing disturbance. The state-and-transition model for the Patagonian steppes. Ecología Austral, 8:191-199.

BESTELMEYER, BT. 2006. Threshold concepts and their use in rangeland management and restoration: The Good, the
Bad, and the Insidious. Restor. Ecol., 14:325-329.

Bestelmeyer, BT; JE Herrick; JR Brown; DA Trujillo \& KM Havstad. 2004. Land management in the American Southwest: a state-and-transition approach to ecosystem complexity. Environ. Manage., 34:38-51.

Bisigato, A; J Ares \& M Bertiller. 2002. Assessment of pristine vegetation structure in semiarid shrublands based on spatial explicit modeling. Phytocoenologia, 32: 581-594.

Bisigato, AJ \& MB Bertiller. 1997. Grazing effects on patchy dryland vegetation in northern Patagonia. J. Arid. Environ., 36:639-653.

Bisigato, AJ \& MB BERTILLER. 2004. Seedling recruitment of perennial grasses in degraded areas of the Patagonian Monte. J. Range Manage., 57:191-196.

Bisigato, A J; MB Bertiller; JO Ares \& Ge Pazos. 2005. Effects of grazing on plant patterns in arid ecosystems of Patagonian Monte. Ecography, 28:561-572.

Bisigato, AJ; RML Laphitz \& AL Carrera. 2008. Nonlinear relationships between grazing pressure and conservation of soil resources in Patagonian Monte shrublands. J. Arid. Environ., 72:1464-1475.

BOWKER, MA. 2007. Biological soil crust rehabilitation in theory and practice: an underexploited opportunity. Restor. Ecol., 15:13-23.

BRISKE, DD; SD FuHLENDORF \& FE SMEINS. 2005. State-andtransition models, thresholds, and rangeland health: a synthesis of ecological concepts and perspectives. Rangeland Ecol. Manag., 58:1-10.

BROWNING, DM \& SR ARCHER. 2011. Protection from livestock fails to deter shrub proliferation in a desert landscape with a history of heavy grazing. Ecol. Appl., 21:1629-1642.

Cabrera, AL. 1971. Fitogeografía de la Republica Argentina. Bol. Soc. Argent. Bot., 16:1-42.

Castellano, MJ \& TJ Valone. 2007. Livestock, soil compaction and water infiltration rate: evaluating a potential desertification recovery mechanism. J. Arid. Environ., 71:97-108.

Cesa, A \& JM Paruelo. 2011. Changes in vegetation structure induced by domestic grazing in Patagonia (Southern Argentina). J. Arid. Environ., 75:1129-1135.

Cipriotti, PA \& MR Aguiar. 2012. Direct and indirect effects of grazing constrain shrub encroachment in semi-arid Patagonian steppes. Appl. Veg. Sci., 15:35-47.

Di Rienzo JA; F Casanoves; MG Balzarini; L Gonzalez; M TABLADA; ET AL. 2009. InfoStat versión 2009. Grupo InfoStat, FCA, Universidad Nacional de Córdoba, Argentina.

DREWRY, JJ. 2006. Natural recovery of soil physical properties from treading damage of pastoral soils in New Zealand and Australia: a review. 2006. Agr. Ecosyst. Environ., 114:159-169.

Evans, RD \& JR Johansen. 1999. Microbiotic crusts and ecosystem processes. Crit. Rev. Plant Sci., 18:183-225.

FuHLENDORF, SD; DD BRISKE \& FE SMEINS. 2001. Herbaceous vegetation change in variable rangeland environments: the relative contribution of grazing and climatic variability. Appl. Veg. Sci., 4:177-188.

Godagnone, RE \& DE Bran. (eds.). 2009. Inventario Integrado de los Recursos Naturales de la Provincia de Río Negro. INTA. Buenos Aires. Pp. 392.

Hillel, D. 1998. Environmental Soil Physics. Academic Press, San Diego. Pp. 771.

JoHNSON, AI. 1991. A field method for measurement of infiltration. U.S. Geological Survey, Water-supply paper 1544-F.

van DE Koppel, J; M RietKerk; F VAN LANGeVelde; L Kumar; 
CA Klausmeier; ET AL. 2002. Spatial heterogeneity and irreversible vegetation change in semiarid grazing systems. Am. Nat., 159:209-218.

KRÖPFL, AI. 1999. Efecto del pastoreo y otros disturbios sobre la estructura y la dinámica de la vegetación de una estepa arbustiva semiárida. Tesis Magister. Universidad de Buenos Aires.

KRÖPfl, AI; GA CeCChI; NM Villasuso \& RA Distel. 2011. Degradation and recovery processes in semi-arid patchy rangelands of northern Patagonia, Argentina. Land. Degrad. Dev., Doi: 10.1002/ldr.1145.

LAYCOCK, WA. 1991. Stable states and thresholds of range condition on North American rangelands: a viewpoint. J. Range Manage., 44:427-433.

Le Houerou, HN. 1989. The grazing land ecosystems of the Africa Sahel. Ecological Studies. Vol. 75. Springer, Berlin. Pp. 282.

Milchunas, DG \& WK Lauenroth. 1993. Quantitative effects of grazing on vegetation and soils over a global range of environments. Ecol. Monogr., 63:328-366.

Milchunas, DG \& I Noy-MeIr. 2002. Grazing refuges, external avoidance of herbivory and plant diversity. Oikos, 99:113-130.

Pazos, GE \& MB Bertiller. 2008. Spatial patterns of the germinable soil seed bank of coexisting perennial-grass species in grazed shrublands of the Patagonian Monte. Plant Ecol., 198:111-120.

Perelman, SB; JCR León \& JP Bussacca. 1997. Floristic changes related to grazing intensity in a Patagonian shrub stepp. Ecography, 20:400-4006.

RADFord, JQ; AF BENNETT \& GJ CheERs. 2005. Landscapelevel thresholds of habitat cover for woodlanddependent birds. Biol. Conserv., 124:317-337.

Read, CF; DH Duncan; PA Vesk \& J Elith. 2011. Surprisingly fast recovery of biological soil crusts following livestock removal in southern Australia. J. Veg. Sci., 22:905-916.

Reynolds, JF; RA Virginia; PR Kemp; AG de Soyza \& DC TREMmel. 1999. Impact of drought on desert shrubs: effects of seasonality and degree of resource island development. Ecol. Monogr., 69:69-106.
RosTAGNO, CM. 1989. Infiltration and sediment production as affected by soil surface conditions in a shrubland of Patagonia, Argentina. J. Range Manage., 42:382-385.

Rostagno, CM \& HF del Valle.1988. Mounds associated with shrubs in aridic soils of northeastern Patagonia: characteristics and probable genesis. Catena, 15:347-359.

SCHEFFer, M;SCARPenter;JA Foley; C FolKe \& B WALKER. 2001. Catastrophic shifts in ecosystems. Nature, 413:591-596.

SePÚlvedA, RB.1999. El infiltrómetro de cilindro simple como método de cálculo de la conductividad hidráulica de los suelos. Experiencias de campo en ámbitos de montañas mediterráneas. Baetica, Estudios de Arte, Geografía e Historia, 21:9-33.

Seymour, CL; SJ Milton; GS Joseph; WRJ Dean; T Ditlhobolo \& GS Cumming. 2010. Twenty years of rest returns grazing potential, but not palatable plant diversity, to Karoo rangeland, South Africa. J. App. Ecol., 47:859-867.

Steffens, M; A Kölbl; KU Totsche \& I Kögel-Knabner. 2008. Grazing effects on soil chemical and physical properties in a semiarid steppe of Inner Mongolia (P.R. China). Geoderma, 143:63-72.

Stringham, TK; WC KRUEGER \& PL SHAVER. 2003. State and transition modeling: An ecological process approach. J. Range Manage., 56:106-113.

Tongway, DJ; AD Sparrow \& MH Friedel. 2003. Degradation and recovery processes in arid grazing lands in central Australia: part 1. Soil and land resources. J. Arid. Environ., 55:310-326.

Thurow, TL; WH BlackBURN \& CA TAYLOR, JR. 1988. Infiltration and interril erosion responses to selected livestock grazing strategies, Edwards Plateau, Texas. J. Range Manage., 41:296-302.

Valone, TJ; M Meyer; JH Brown \& RM Chew. 2002. Timescale of perennial grass recovery in a desertified arid grasslands following livestock removal. Conserv. Biol., 16:995-1002.

Verbist, K; S TORFS; WM CORNelis; R OYARZÚN; G SOTO; ET AL. 2010. Comparison of single- and double-ring infiltrometer methods on stony soils. Vadose Zone J., 9:462-475. 


\section{INFORMACIÓN SUPLEMENTARIA}

\section{SUPPLEMENTARY INFORMATION}

Vista general del área de estudio. Las áreas clausuradas (AC) (10 años de antigüedad) se muestran a la izquierda, mientras que las áreas pastoreadas (AP) se encuentran a la derecha.

Overview of the area of study. The ungrazed areas (AC) (10 years excluded) are shown on the left whereas the grazed areas (AP) are shown on the right.

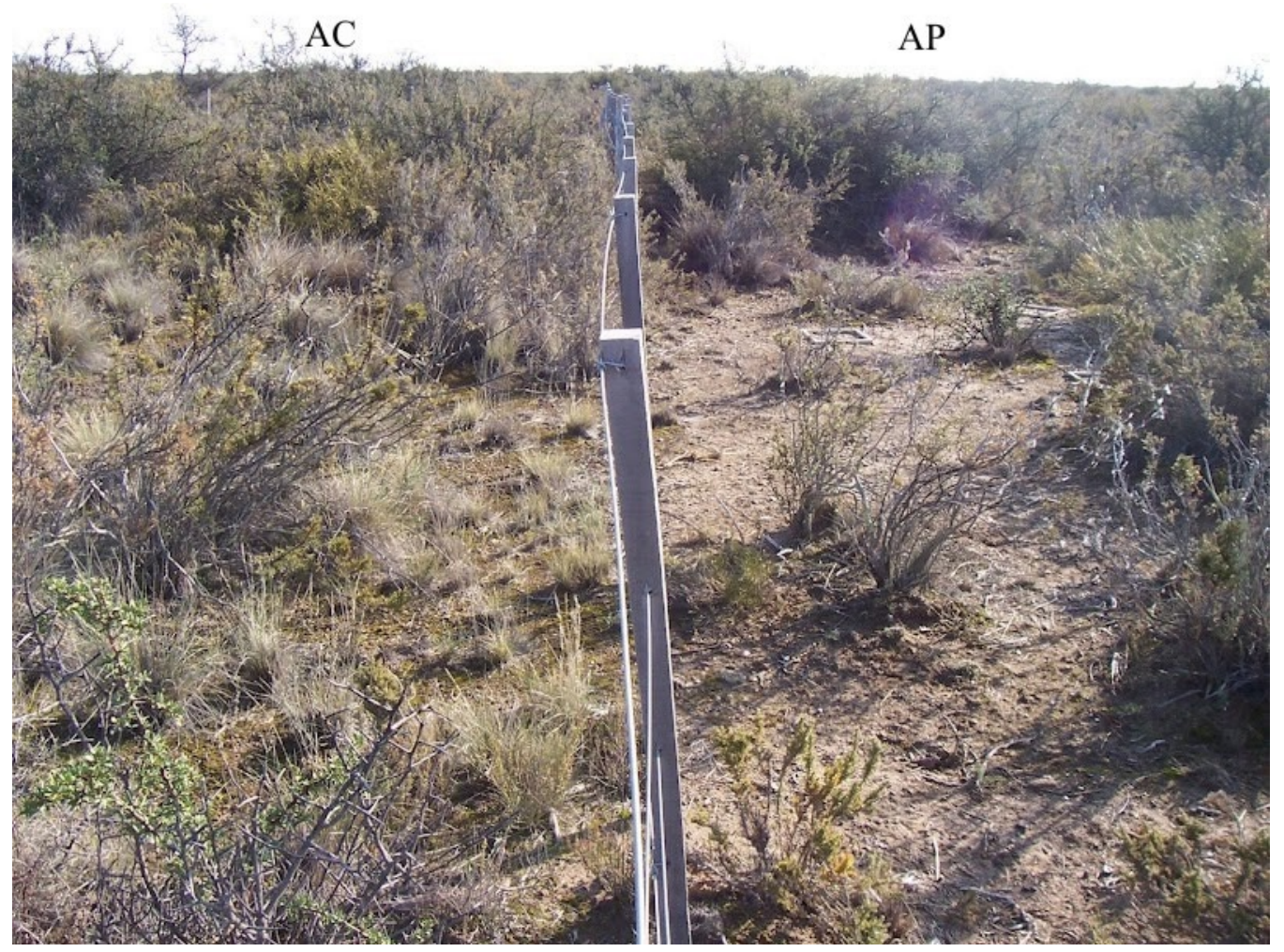

